\title{
EL DERECHO DE AUTOR EN EL TPP DESDE UNA PERSPECTIVA
}

EDITORIAL

\section{Quetzalli de la Concha Pichardo \\ UniVERSIDAd NACIONAL AutónOMa de MÉXICo}

Especialista en Propiedad Intelectual de la Facultad de Derecho de la UNAM (México). Vicepresidenta del Centro Mexicano de Protección y Fomento de los Derechos de Autor México. Gerente de Derechos de Autor de PenguinRandomHouse Grupo Editorial.

En este artículo se presenta el contexto y antecedentes del TPP en materia de Derechos de Autor así como un análisis de las implicaciones de éste. De igual manera se da un punto de vista sobre el papel de los Estados Unidos en este tratado y se comentan los puntos más relevantes del TPP en relación al Derecho de Autor y los Derechos conexos. Entre los cambios más importantes destacan la ampliación del periodo de protección a un plazo mínimo de 70 años, el reconocimiento equivalente de la naturaleza exclusiva del Derecho de Autor y de los derechos de los artistas intérpretes y productores de fonogramas. El tratado reconoce limitaciones y excepciones que tienen el objetivo de equilibrar el sistema de Derecho de Autor; introduce medidas tecnológicas de protección e información sobre la gestión de derechos así como procedimientos y recursos civiles, administrativos y penales para reprimir actividades que infringen derechos de autor o conexos. Por último se propone un sistema más agresivo de colaboración de los prestadores de servicios en Internet ISP que tiene un 


\section{EL DERECHO DE AUTOR EN EL TPP DESDE UNA PERSPECTIVA EDITORIAL}

efecto directo sobre los usuarios.

El Acuerdo estratégico Transpacífico de asociación económica (TPP) es actualmente el tratado más importante en materia de propiedad intelectual (PI), a la cual le dedica por entero su capítulo número 18. En este artículo se aborda en específico lo que se refiere al Derecho de Autor y Derechos conexos, los cuales están previstos en la sección $\mathrm{H}$, que va del artículo 18.57 al 18.70 , aunque por supuesto también les son aplicables las disposiciones generales del capítulo, así como las que se refieren a los procedimientos civiles, administrativos y penales ante infracciones de derechos de propiedad intelectual.

Debido a que el TPP se mantuvo internacionalmente fuera del debate público, que sus negociaciones fueron a puerta cerrada y sus textos se conservaron en secreto existe un halo de incertidumbre alrededor del tratado ${ }^{1}$.

\section{CONTEXTO DEL TPP}

Antes de abordar el contenido del TPP, es importante contextualizar brevemente el entorno digital y nacional de este tratado, así como los antecedentes normativos del mismo.

\section{ERA DigitaL}

Nos encontramos en una era denominada digital, pues la comunicación, transmisión e intercambio de contenidos se llevan a cabo a través de procesos y medios digitales, generando una transición de los medios analógicos (entorno en el cual se crearon las bases del derecho de autor) a los digitales. Esto genera la necesidad de

\footnotetext{
${ }^{1}$ González Morgado Alejandro, El camino incierto del TPP, texto incluido en La política exterior en México: metas y obstáculos coordinado por Guadalupe González y Olga Pellicer, México, siglo XXI editores, 2013, pp. 229- 234.
} 
responder con una regulación efectiva a nuevos supuestos de uso, géneros de obras y mecanismos de divulgación.

La tecnología digital ha producido un gran efecto que consiste en la convergencia entre las fronteras tradicionales, los medios de difusión o tecnologías y los distintos soportes clásicos de las obras como pudieran ser los libros, los discos, las cintas de video, etc. Estas fronteras se difuminan para convertirse en un solo soporte universal.

Asimismo, este fenómeno afecta la mentalidad de la sociedad, actualmente, la importancia del libro y su portada, del disco y su funda, que en otras generaciones motivaba o incitaba a la adquisición de la obra y enriquecía el placer de su posesión, parecen menos importantes que en el pasado. Ahora, el contenido es lo principal, y las generaciones más jóvenes no ven ningún atentado al derecho de autor ligado a la obra musical, escrita o gráfica, en el hecho de almacenarla en cualquier soporte digital privado.

Entre los principales retos que se presentan en el entorno digital podemos resaltar los siguientes:

a) Participantes de la cadena de comunicación. Creador o titular de la obra, prestador de servicios de transmisión en Internet, host de contenido y usuario(s).

b) Eliminación de fronteras. Los contenidos se pueden compartir o transmitir en cuestión de segundos a lugares diferentes sin importar las distancias.

c) Prescindir de ejemplares. El usuario puede obtener una obra descargándola desde su ordenador o bien accediendo a ella en streaming sin necesidad de descargarla en una copia permanente en su equipo. 


\section{EL DERECHO DE AUTOR EN EL TPP DESDE UNA PERSPECTIVA EDITORIAL}

d) Formatos distintos y con posibilidad de ser compuestos por varias obras.

Este es el caso de las obras multimedia.

e) Mayor accesibilidad de los usuarios a recursos tecnológicos.

f) Falta de recursos legales para suspender actividades infractoras.

Tradicionalmente, las prácticas contractuales estaban orientadas al mercado analógico. En el caso de la práctica editorial, los contratos se orientaban a la reproducción de ejemplares, así como a la distribución y comercialización de los mismos. Entre 2011 y 2012 el fenómeno de ventas de ejemplares digitales de las editoriales de lengua inglesa generó una reacción importante por parte de las editoriales en México, ya que de la noche a la mañana el ebook había alcanzado una marca histórica de $30 \%$ del mercado de libros en Estados Unidos. Por lo tanto, los derechos enfocados en el mercado digital comenzaron a formar parte de los contratos.

En México, el libro digital todavía representa una parte muy pequeña pues ronda sólo el $5 \%^{2}$ del mercado en general; no obstante, las herramientas digitales han generado dos fenómenos importantes ${ }^{3}$ :

1) Robo de archivos digitales de los libros editados, lo cual ha potenciado la producción de libros piratas a una escala nunca vista:

- $\quad$ En 2012, tres de cada diez libros que se vendieron en México fueron piratas.

- $\quad$ En 2015, cerca de 45 millones de libros piratas circularon en el país y en Centroamérica.

\footnotetext{
${ }^{2}$ Conforme al análisis de la Cámara Nacional de la Industria Editorial realizado en 2014 y publicado en: http://www.dosdoce.com/wp-content/uploads/2015/09/An-overview-of-the-digital-evolution-of-theMexican-Publishing-Market.pdf

${ }^{3}$ Publicado por el Centro Mexicano de Protección y Fomento de los Derechos de Autor, información disponible en http://cempro.com.mx/sitio/
} 
- Esta circulación representa pérdidas económicas de cerca de 2 mil millones de pesos al año.

2) Descarga ilegal de libros: En el año 2012 se registraron 88 millones de descargas ilegales de libros por Internet.

\section{ANTECEDENTES DEL TPP EN MATERIA DE DERECHO DE AUTOR}

México forma parte de dos importantes tratados en materia de comercio, los cuales incluyen estándares internacionales para la protección de la propiedad intelectual y conforman la segunda generación de tratados de $\mathrm{PI}^{4}$ :

ADPIC. El Acuerdo sobre Aspectos de los Derechos de Propiedad Intelectual relacionados con el Comercio, cuyo objetivo fundamental es fomentar una protección eficaz y adecuada de los derechos de PI y fungir como puente a otros cuerpos normativos, como el Convenio de Berna y el Convenio de Roma, basándose en principios de trato nacional y de la nación más favorecida. Estableció principios básicos sobre la PI tendientes a armonizar sistemas entre los países firmantes y requisitos mínimos para la protección de los titulares de derechos de PI.

TLCAN. El Tratado de Libre Comercio de América del Norte, que tiene entre sus objetivos proveer de acciones efectivas y expeditas de carácter civil y penal para los nacionales de los tres países que forman parte del acuerdo.

Ambos tratados son de carácter comercial y vinculan a mecanismos de solución de controversias, toman como base los tratados que tienen como eje fundamental las convenciones de París y Berna, aumentan la protección de la PI, establecen normas para

\footnotetext{
${ }^{4}$ Becerra Ramírez, Manuel, La Propiedad Intelectual en transformación, México, Editorial Porrúa, 2009, p.18- 25 .
} 


\section{EL DERECHO DE AUTOR EN EL TPP DESDE UNA PERSPECTIVA EDITORIAL}

ser incorporadas en el derecho interno y se ligan a sanciones comerciales en caso de incumplimiento.

Sobre la regulación de responsabilidad de Prestadores de Servicios en Internet (ISP, por sus siglas en inglés) en la experiencia internacional, se han promulgado leyes que han marcado antecedentes importantes, como la Digital Millenium Copyright Act (DMCA) (EUA 1998), Ley Hadopi (tres pasos) para la promoción de la difusión de obras y protección de derechos de creadores en internet (Francia 2010), Ley de economía sostenible en su disposición final $43^{\circ}$ para la regulación de descargas en internet (España 2010), y la EUCD o directiva de la Unión Europea sobre Derecho de Autor (UE 2001 *limitaciones a la copia privada en internet), que basado en el Tratado sobre Derecho de Autor de la OMPI (WPPT 1996), cuyo objetivo principal es la armonización del régimen de Propiedad Intelectual y la protección de obras en el entorno digital.

\section{El PAPEL DE ESTADOS Unidos}

Considerando que los Estados Unidos juegan un papel primordial tanto en las negociaciones del TPP como en los parámetros aceptados en la versión final, cabe mencionar que su gobierno tiene clara la necesidad de proteger los intereses de las compañías de contenidos, las cuales representan un importante sector de su economía. Es por ello que ha ejercido una aproximación vertical referenciando sus leyes como eje legal en relaciones bilaterales de comercio. La DMCA es la base del modelo legal que Estados Unidos exporta en sus tratados; entre sus líneas principales se encuentran el incremento sustancial de penas por infracción de derechos de autor, sanciones por la 
elusión de medidas tecnológicas de protección (MTP) y mecanismos de control de contenidos en internet a petición de los titulares, con la colaboración de los ISPs.

Respecto de la regulación de responsabilidad de los ISPs, el TPP representa uno de los varios intentos de Estados Unidos para endurecer las medidas de control no sólo para los prestadores de servicios, sino para los usuarios de internet. SOPA (Stop Online PiracyAct) y PIPA (Protect Intelectual PropertyAct) son los documentos que se intentaron aplicar anteriormente, pero en ambos casos no fue posible su entrada en vigor debido a la impopularidad de sus disposiciones y al rechazo público de sus ciudadanos, sin embargo, varias de éstas se encuentran presentes en el $\mathrm{TPP}^{5}$. Esto no debería sorprendernos, considerando que bajo el argumento de ser un tratado comercial, sus términos se negociaron y definieron en total secrecía y por tanto no existió participación alguna de la sociedad.

\section{TEMAS ReleVANTES DEL TPP EN DERECHO DE AUtOR Y DERECHOS CONEXOS}

Algunos de los temas que sobresalen en el TPP para el Derecho de Autor y Derechos Conexos son:

1. Incremento en el periodo de protección. Ampliando el plazo mínimo a 70 años.

Éste es un cambio importante si consideramos que el Convenio de Berna estipula 50 años, el cual ha sido reconocido tradicionalmente como el plazo mínimo de

\footnotetext{
5 Botero, Carolina y Gaitán, César, El Acuerdo Estratégico Transpacífico de Asociación Económica (TPP): una visión regional del derecho de autor, incluido en El Acuerdo de Asociación Transpacífico (TPP): ¿bisagra o confrontación entre el Atlántico y el Pacífico?, coordinador Oropeza García Arturo, México, Universidad Nacional Autónoma de México/Instituto de Investigaciones Jurídicas, 2013, pp.341346.
} 


\section{EL DERECHO DE AUTOR EN EL TPP DESDE UNA PERSPECTIVA EDITORIAL}

protección de estos derechos. En el caso de México, éste no afecta a autores, pero sí a los titulares de derechos conexos (cinco años más).

Con respecto a la pregunta sobre si la ampliación del plazo beneficia a los autores o afecta el acceso a la cultura, existen algunos puntos a considerar:

- La ampliación del plazo desconoce el valor del dominio público para favorecer una sociedad creativa e innovadora.

- Genera interrogantes sobre el equilibrio entre una remuneración justa para el autor o titular de derechos y el acceso a la cultura (artículo $4^{\circ}$ constitucional).

- Es una decisión basada en un principio de "mayor plazo, mayor beneficio". Es difícil pensar que esta ampliación tenga un efecto positivo para los causahabientes del autor o titular de derechos conexos, pero en definitiva es una garantía importante para las productoras y empresas de contenidos.

Es preciso mencionar que la entrada de obras al dominio público genera un mayor acceso de la población a contenidos creativos y deja abierta la posibilidad de generar contenidos protegidos como obras derivadas. Ejemplo de esto es que las editoriales buscan obtener derechos exclusivos en el caso de obras nuevas o inéditas, pero tratándose de aquellas que son de dominio público, la competencia consiste en una buena edición, mejores traducciones y precios accesibles.

No se debe perder de vista que la contraposición de los intereses del autor y del público sólo es aparente, pues así como el público está interesado en acceder a las obras, también el autor quiere que se difundan con la mayor amplitud. 
2. Reconocimiento equivalente de la naturaleza exclusiva del Derecho de Autor y de los derechos de los artistas intérpretes o ejecutantes, y productores de fonogramas.

En su artículo 18.61 el TPP puntualiza que no existe jerarquía entre estos derechos en el caso de autorizaciones sobre fonogramas.

Este reconocimiento equivale a consentir la tendencia norteamericana de identificar los derechos de los autores de las obras con los derechos conexos, difuminando la naturaleza propia del Derecho de Autor en que la actividad creativa genera un vínculo único entre el autor y su obra, por tanto una serie de facultades que sólo son inherentes a la persona física que creó la obra, incluyendo el plazo de protección, son idénticas para un titular de derechos conexos que bien puede ser una productora musical, un editor o un productor cinematográfico. Equiparar la naturaleza de los derechos del autor con los derechos conexos es reconocer que la titularidad es lo que impera para ejercer facultades de autorizar o prohibir el uso y goce de las obras lo cual es un principio ajustado al sistema de copyright.

Lo anterior se suma a una tendencia en la legislación nacional de presunción de cesión de los derechos del autor en favor de los productores y en general de obras realizadas por encargo, con la excepción concreta de las obras musicales incorporadas a una obra audiovisual, y que omite obras tan importantes para las producciones audiovisuales como las obras literarias (argumento, libro, guion, etc.) Al respecto Delia 


\section{EL DERECHO DE AUTOR EN EL TPP DESDE UNA PERSPECTIVA EDITORIAL}

Lipszyc advierte que esto configura lo que se denomina "un derecho de autor sin autor ${ }^{\prime \prime 6}$.

\section{Limitaciones y excepciones de los derechos de explotación.}

Para la limitación o excepción de derechos patrimoniales, mantiene la regla de los tres pasos:

1) Casos especiales o excepciones.

2) Que no atente contra la normal explotación de la obra.

3) Que no genere un daño injustificado a los intereses del autor o titular.

En este sentido el tratado incluye un artículo en el cual dispone que las partes procurarán alcanzar un equilibrio en su sistema de derecho de autor y derechos conexos, mediante limitaciones y excepciones que incluyan el entorno digital y consideren fines legítimos como crítica, cobertura de noticias, investigación y enseñanza, así como facilitar el acceso a personas invidentes, con discapacidad visual o con otras dificultades para la lectura. Este acceso tiene como base lo dispuesto en el Tratado de Marrakech para facilitar el acceso a las obras publicadas a las personas ciegas, con discapacidad visual o con otras dificultades para acceder al texto impreso, del que México también forma parte y que generó una de las más recientes reformas en nuestra LFDA, adicionando una excepción al artículo 148, misma que tiene una deficiente redacción pues no regula de manera concreta los supuestos del Tratado de Marrakech y deja abierta la excepción a cualquier tipo de discapacidad lo cual ha generado que autores y editoriales sean afectados por ediciones y adaptaciones no autorizadas que circulan en la

${ }^{6}$ Lipszyc, Delia, Los autores y el derecho, en Un encuentro no casual: cultura, ciencias económicas y derecho, coordinado por Asuaga Carolina, Montevideo, fundación Cultura Universitaria, 2009, pp. 106107 
red, bajo el argumento de que se encuentran amparadas en la nueva excepción del artículo 148 de la LFDA.

El equilibrio en el sistema del derecho de autor y derechos conexos tiene una estrecha relación con el derecho a la cultura que al igual que sucede con el derecho a la información se trata de una relación que Eduardo de la Parra ${ }^{7}$ considera paradójica, en la medida que estas figuras se apoyan y fortalecen mutuamente pero pueden llegar a colisionar en ciertas circunstancias. Lo que es un hecho es que tanto el derecho de autor como el derecho a la cultura persiguen los mismos propósitos y esto es reconocido en el artículo $1^{\circ}$ de la LFDA que reconoce como objeto "la salvaguarda y promoción del acervo cultural de la nación". Por una parte los derechos morales preservan el patrimonio cultural, ayudan a respetar la verdad histórica y son un aliciente de la creatividad; en el caso de los derechos de explotación se traducen en incentivos para la creación de cultura.

\section{Medidas tecnológicas de protección e información sobre la gestión de} derechos.

Las medidas tecnológicas de protección (MTP) son aquellas tecnologías que permiten controlar el acceso a contenidos protegidos; por ejemplo, la regionalización de ejemplares de obras audiovisuales que ayudan a restringir la reproducción en países o zonas distintas a las que se dirigió la venta del producto.

En el caso de los ebooks, el Digital Rights Management (DRM por sus siglas en inglés) es la medida tecnológica más utilizada para controlar el acceso y descarga de

\footnotetext{
${ }^{7}$ De la Parra, Eduardo, Derechos humanos y Derechos de autor, las restricciones al derecho de explotación, México, Universidad Nacional Autónoma de México/Instituto de Investigaciones Jurídicas, 2015, p.589.
} 


\section{EL DERECHO DE AUTOR EN EL TPP DESDE UNA PERSPECTIVA EDITORIAL}

libros digitales y que consiste en una especie de autenticación entre el usuario que adquirió el contenido, en algunos casos el reconocimiento del equipo en el que lo abrió y el contenido mismo. Es importante señalar que desde hace años la utilización de medidas tecnológicas de protección es un requerimiento obligatorio de la mayoría de las agencias literarias en los contratos de edición.

El TPP estipula que las partes deben proporcionar protección legal adecuada y recursos legales efectivos contra la elusión de las medidas tecnológicas que utilizan los autores o titulares de derechos conexos para controlar el acceso a contenidos protegidos.

Respecto de estas medidas Eduardo de la Parra $^{8}$ advierte entre los inconvenientes que presentan las MTP la posibilidad de ser un mecanismo para controlar e impedir la difusión de obras del dominio público, controlar la comunicación privada de obras o impedir que el usuario realice alguna conducta permitida por una restricción al derecho de explotación, lo cual es contrario al derecho a la información y rompe el equilibrio entre los derechos de los autores y los derechos de los usuarios.

La información sobre la gestión de derechos (IGD) son los datos que identifican una obra, al autor de ésta o al titular de cualquier derecho sobre la misma, así como la información sobre los términos y condiciones de utilización de la obra.

Aunque el tratado especifica que no se obliga a las partes a exigir al titular del derecho que incorpore IGD a las copias de su obra, en el mercado de libros en formato digital proporcionar esta información, junto con los archivos, es un requisito indispensable de las plataformas de internet para administrar su catálogo y ventas, así como facilitar la búsqueda de los usuarios.

\footnotetext{
${ }^{8}$ Ibídem, pp.651- 655.
} 
5. Procedimientos y recursos civiles, administrativos y penales para reprimir actividades que infringen derechos de autor o conexos.

En esta parte del tratado resaltan los siguientes lineamientos:

- $\quad$ Obligación de las partes para establecer medidas eficaces en contra de cualquier acción infractora, incluidas las realizadas en el entorno digital.

- $\quad$ Presunción de titularidad de derechos de autor y conexos. Salvo prueba en contrario, se reconocerá como autor o titular si su nombre es indicado de manera usual con esa calidad.

- $\quad$ Facultar a sus autoridades para imponer medidas precautorias, entre ellas el aseguramiento de dispositivos y productos involucrados a una actividad prohibida, incluyendo las relativas a las MTP e IGD, ordenar indemnizaciones, pago de gastos y costas procesales, así como la destrucción de dispositivos y productos.

Por supuesto los usuarios pueden ser sujetos activos de este tipo de infracciones en internet, por ejemplo, la utilización de servidores Proxy para evadir bloqueos regionales en descargas o streaming, o la remoción del DRM de una obra protegida a través de herramientas informáticas diseñadas para ello.

6.Sistema más agresivo de colaboración de los prestadores de servicios en internet ISP:

Se contemplan varios mecanismos para incrementar la participación de los ISPs

1. Establecimiento de puertos seguros. Se considerará que un ISP cuenta con un puerto seguro cuando cumpla con los mecanismos de disuasión del almacenamiento no autorizado y la transmisión de materiales protegidos.

2. Incentivos legales para cooperar en la disuasión del almacenamiento no autorizado o transmisión de materiales protegidos. Son incentivos relacionados con la 


\section{EL DERECHO DE AUTOR EN EL TPP DESDE UNA PERSPECTIVA EDITORIAL}

exoneración de responsabilidad para los intermediarios (ISP) por las presuntas infracciones al DA que cometan sus usuarios en Internet.

3. Obligación de bloquear o remover contenidos infractores alojados en sus sistemas o redes a petición del titular de derechos de autor o derechos conexos, lo cual se define como un mecanismo de retiro privado. El antecedente de este mecanismo es la DMCA, esquema bajo el cual las editoriales han trabajado desde hace años porque es el que reconocen y aplican la mayoría de los PSI de las plataformas principales, como Amazon o Google (YouTube), a través de notificaciones directas a los ISPs o de plataformas como Atributtor o Rightsguardian, dedicadas al monitoreo de usos ilegales en Internet y que envían notificaciones de forma automatizada.

4. $\quad$ Ausencia de responsabilidad para ISPs por remoción de contenidos de buena fe.

5. Observancia de principios de debido proceso y de privacidad al proporcionar al titular de DA información del presunto infractor. Es indispensable que las disposiciones de aplicación a través de procedimientos judiciales o administrativos garanticen el derecho a la intimidad de las personas y la protección de sus datos personales.

\section{CONCLUSIONES}

1. El TPP es un tratado celebrado en un contexto de asimetrías entre las partes respecto de su capacidad económica y poder a nivel internacional, en Derechos de Autor y conexos es marcada la intervención de Estados Unidos, la cual responde a los intereses de las compañías establecidas en esa nación. Dichas asimetrías pueden 
generar una clara desigualdad en la aplicación de las disposiciones del tratado y sus efectos en cada país, incluido México.

2. Es importante plantearse tanto en el TPP como en la legislación mexicana si el incremento en el plazo de protección realmente genera un beneficio para los autores o si prevalece una afectación social por el aplazamiento de la entrada de contenido creativo al dominio público.

3. El entorno digital presenta la necesidad de regular a los PSI, considerando su importancia como participantes en la cadena de comunicación de contenidos. Sin embargo, no debemos perder de vista que dicha regulación tiene un efecto directo sobre los usuarios, por lo que debe atender a procesos que garanticen el derecho a la intimidad de las personas y protección de sus datos personales.

\section{REFERENCIAS BIBLIOGRÁFICAS}

Becerra Ramírez, M. (2009). La Propiedad Intelectual en transformación. México: Editorial Porrúa.

Botero, C. y Gaitán, C. (2013). El Acuerdo Estratégico Transpacífico de Asociación Económica (TPP): una visión regional del derecho de autor. En Oropeza García, A. (Coord.), El Acuerdo de Asociación Transpacífico (TPP): ¿bisagra o confrontación entre el Atlántico y el Pacífico? México: Universidad Nacional Autónoma de México/Instituto de Investigaciones Jurídicas.

De la Parra, E. (2015). Derechos humanos y Derechos de autor, las restricciones al derecho de explotación. México: Universidad Nacional Autónoma de México/Instituto de Investigaciones Jurídicas. 
Lipszyc, D. (2009). Los autores y el derecho. En Asuaga, C. (Coord.), Un encuentro no casual: cultura, ciencias económicas y derecho. Montevideo: Fundación Cultura Universitaria.

González Morgado, A. (2013). El camino incierto del TPP. En González, G. y Pellicer, O. (Coords.), La política exterior en México: metas y obstáculos. México: Siglo XXI editores.

\section{Publicaciones En internet}

Análisis de la Cámara Nacional de la Industria Editorial realizado en 2014 publicado en: http://www.dosdoce.com/wpñ-content/uploads/2015/09/An-overview-of-thedigital-evolution-of-the-Mexican-Publishing-Market.pdf

Publicado por el Centro Mexicano de Protección y Fomento de los Derechos de Autor, información disponible en: http://cempro.com.mx/sitio/ 